\title{
Demonstration of periodic nanostructure formation with less ablation by double-pulse laser irradiation on titanium
}

\section{$\operatorname{AUTHOR}(\mathrm{S}):$}

Furukawa, Yuki; Sakata, Ryoichi; Konishi, Kazuki; Ono, Koki; Matsuoka, Shusaku; Watanabe, Kota; Inoue, Shunsuke; Hashida, Masaki; Sakabe, Shuji

\section{CITATION:}

Furukawa, Yuki ...[et al]. Demonstration of periodic nanostructure formation with less ablation by double-pulse laser irradiation on titanium. Applied Physics Letters 2016, 108(26): 264101.

\section{ISSUE DATE:}

2016-06-27

URL:

http://hdl.handle.net/2433/267217

\section{RIGHT:}

(c) 2016 Author(s). Published by AlP Publishing.; This article may be downloaded for personal use only. Any other use requires prior permission of the author and AIP Publishing. This article appeared in Yuki Furukawa, Ryoichi Sakata, Kazuki Konishi, Koki Ono, Shusaku Matsuoka, Kota Watanabe, Shunsuke Inoue, Masaki Hashida, and Shuji Sakabe, "Demonstration of periodic nanostructure formation with less ablation by double-pulse laser irradiation on titanium", Appl. Phys. Lett. 108, 264101 (2016) and may be found at https://doi.org/10.1063/1.4955035. 


\section{Demonstration of periodic nanostructure} formation with less ablation by double-pulse laser irradiation on titanium

Cite as: Appl. Phys. Lett. 108, 264101 (2016); https://doi.org/10.1063/1.4955035

Submitted: 26 April 2016 • Accepted: 18 June 2016 • Published Online: 30 June 2016

(iD) Yuki Furukawa, Ryoichi Sakata, Kazuki Konishi, et al.
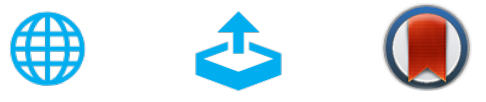

\section{ARTICLES YOU MAY BE INTERESTED IN}

Femtosecond laser-induced periodic surface structures

Journal of Laser Applications 24, 042006 (2012); https://doi.org/10.2351/1.4712658

Formation of controllable 1D and 2D periodic surface structures on cobalt by femtosecond double pulse laser irradiation

Applied Physics Letters 115, 031601 (2019); https://doi.org/10.1063/1.5103216

On the role of surface plasmon polaritons in the formation of laser-induced periodic surface structures upon irradiation of silicon by femtosecond-laser pulses

Journal of Applied Physics 106, 104910 (2009); https://doi.org/10.1063/1.3261734

母QBLOX

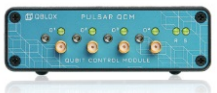

1 qubit
Shorten Setup Time Auto-Calibration More Qubits

Fully-integrated Quantum Control Stacks Ultrastable DC to $18.5 \mathrm{GHz}$ Synchronized $<<1$ ns Ultralow noise

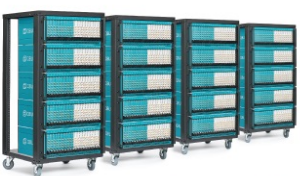

100s qubits

visit our website > 


\title{
Demonstration of periodic nanostructure formation with less ablation by double-pulse laser irradiation on titanium
}

\author{
Yuki Furukawa, ${ }^{1,2,3}$ Ryoichi Sakata, ${ }^{1,2}$ Kazuki Konishi, ${ }^{1,2}$ Koki Ono, ${ }^{1,2}$ Shusaku Matsuoka, ${ }^{1}$ \\ Kota Watanabe, ${ }^{2,3}$ Shunsuke Inoue,,${ }^{2,3}$ Masaki Hashida, ${ }^{2,3}$ and Shuji Sakabe ${ }^{2,3}$ \\ ${ }^{1}$ Faculty of Science, Kyoto University, Kitashirakawa, Sakyo, Kyoto 606-8502, Japan \\ ${ }^{2}$ Graduate School of Science, Kyoto University, Kitashirakawa, Sakyo, Kyoto 606-8502, Japan \\ ${ }^{3}$ Advanced Research Center for Beam Science, Institute for Chemical Research, Kyoto University, Gokasho, \\ Uji, Kyoto 611-0011, Japan
}

(Received 26 April 2016; accepted 18 June 2016; published online 30 June 2016)

\begin{abstract}
By pairing femtosecond laser pulses (duration $\sim 40 \mathrm{fs}$ and central wavelength $\sim 810 \mathrm{~nm}$ ) at an appropriate time interval, a laser-induced periodic surface structure (LIPSS) is formed with much less ablation than one formed with a single pulse. On a titanium plate, a pair of laser pulses with fluences of 70 and $140 \mathrm{~mJ} / \mathrm{cm}^{2}$ and a rather large time interval ( $\left.>10 \mathrm{ps}\right)$ creates a LIPSS with an interspace of $600 \mathrm{~nm}$, the same as that formed by a single pulse of $210 \mathrm{~mJ} / \mathrm{cm}^{2}$, while the double pulse ablates only $4 \mathrm{~nm}$, a quarter of the ablation depth of a single pulse. Published by AIP Publishing. [http://dx.doi.org/10.1063/1.4955035]
\end{abstract}

Nanostructures produced by femtosecond laser irradiation are being actively studied as a result of recent progress in femtosecond laser technology. The formation of laserinduced periodic surface structure (LIPSS) has been demonstrated with femtosecond lasers for metals, semiconductors, and insulators. ${ }^{1-3}$ A femtosecond LIPSS (hereinafter, simply LIPSS) is characterized by a periodic structure interspace shorter than the laser wavelength and a direction perpendicular to the laser polarization. It has been confirmed experimentally that the laser fluence for LIPSS formation $\left(F_{\text {LIPSS }}\right)$ is bounded $\left(F_{\mathrm{th}}<F_{\text {LIPSS }}<F_{\mathrm{M}}\right)$ where the minimum is around the ablation threshold $\left(F_{\mathrm{th}}\right)$ and $F_{\mathrm{M}}$ is the maximum fluence for which the periodic nanostructure is formed. ${ }^{4}$ However, the detailed physical mechanism of the LIPSS phenomenon and this fluence range are still debated and a systematic experimental database has not yet been completed. Interest in LIPSS is increasing from the viewpoint of its applications to laser micro-processing, such as friction reduction for metals, ${ }^{5,6}$ hydrophobicity enhancement of solid surfaces, ${ }^{7}$ improvement of osteoblast biocompatibility, ${ }^{8}$ and structural coloration of metals. ${ }^{9,10}$ It is desirable for such applications that the interspace and the ablation depth of the LIPSS can be controlled separately. Both LIPSS formation and the ablation rate depend on the surface state, ${ }^{11,12}$ so if the surface state can be changed by a laser pulse, the irradiation of a double pulse with an appropriate interpulse interval $\Delta t$ would have an effect different from that of singlepulse irradiation. For double-pulse irradiation, the ablation rate in the fluence range above $F_{\mathrm{M}}$ (for $\Delta t$ intervals up to $3 \mathrm{~ns})^{13}$ and LIPSS formation in the fluence range of $F_{\text {th }} \sim F_{\mathrm{M}}$ (for $\Delta t$ intervals up to several hundred picoseconds $)^{14-16}$ have been studied individually. However, the relation between LIPSS formation and ablation rate has not been studied systematically. To discuss the potential of femtosecond laser nano-processing by double-pulse laser irradiation, it is important to investigate the LIPSS and ablation properties in detail, which is the purpose of this study.
In the experiment of double-pulse irradiation on metal, linearly polarized laser pulses are delivered from the $\mathrm{T}^{6} \mathrm{Ti}$ sapphire laser system (ICR, Kyoto University; $810 \mathrm{~nm}$ central wavelength, 40 fs pulse duration, $10 \mathrm{~Hz}$ repetition rate). A single pulse is divided into two pulses by a beam splitter. To control the interval between the first and second pulses, a retroreflector on a translational stage (working length $300 \mathrm{~mm}$ ) is placed in the optical path of the second pulse beam. The two pulses are aligned collinearly so their polarizations are parallel. A titanium (Ti) plate of $99.5 \%$ purity is used as a target. The roughness of the plate, as characterized by the arithmetic mean value, is less than $2 \mathrm{~nm}$. Before the experiment, the polished titanium plate is cleaned with an ultrasonic cleaner (USK-2R, AS ONE, Inc.) in acetone of $99.5 \%$ purity for $20 \mathrm{~min}$. The double pulse is focused on the Ti surface with a quartz lens (focal length $f=300 \mathrm{~mm}$ ). The laser spot diameter (FWHM) on the target is $90 \mu \mathrm{m}$. The irradiation is performed in air at 1 bar pressure. Table I shows the experimental irradiation parameters. The laser fluence is obtained by measuring the laser energy with a pyroelectric detector and the spot size with a CCD camera. The pulse beam profile is Gaussian and the laser fluence is the maximal fluence on the laser beam axis. The pulse energy is controlled by an energy reducer in each beam path. The first pulse fluence is set to $F_{1}=70 \mathrm{~mJ} / \mathrm{cm}^{2}$ or $140 \mathrm{~mJ} / \mathrm{cm}^{2}$ while keeping the second pulse fluence at $F_{2}=140 \mathrm{~mJ} / \mathrm{cm}^{2}$. The ablation threshold fluence $F_{\text {th }}$ for the Ti plate is observed to be $F_{\mathrm{th}} \sim 100 \mathrm{~mJ} / \mathrm{cm}^{2}$, and we set $F_{1}$ below and above $F_{\mathrm{th}}$, respectively. For simplicity, hereafter, we denote the fluences of double pulses by pairs of numbers $\left(F_{1}\left[\mathrm{~mJ} / \mathrm{cm}^{2}\right]\right.$, $\left.F_{2}\left[\mathrm{~mJ} / \mathrm{cm}^{2}\right]\right)$, such as $(70,140)$. To measure the LIPSS

TABLE I. Irradiation parameters.

\begin{tabular}{lcc}
\hline \hline Fluence $\left(\mathrm{mJ} / \mathrm{cm}^{2}\right)$ & $F_{1}$ & 70,140 \\
& $F_{2}$ & 140 \\
Time interval (ps) & $\Delta t$ & $0.16,1,4,8,10,12,14,20,40$, \\
& & $80,160,320,640,1280$ \\
\hline \hline
\end{tabular}


interspace and the ablation rate accurately, the number of pulse pairs was 50 and 200, respectively. The accuracy of intervals $\Delta t$ between double pulses is \pm 6.6 fs. Single-pulse irradiation is also done with fluences of 140, 210, and $280 \mathrm{~mJ} / \mathrm{cm}^{2}$.

The LIPSS and ablation rate are analyzed as follows. Laser-produced craters on the titanium plate are imaged with a scanning electron microscope (SEM; JSM-5560, JEOL), and the periodicity of structures is evaluated from the $1 \mathrm{D}$ Fourier transformation spectrum of each SEM image. We observe only the center part of images $(16.7 \times 16.7 \mu \mathrm{m})$, in which the laser intensity is constant to within $5 \%$. The ablation rate is defined as crater depth per pulse (for a single pulse) or per pair of pulses (for a double pulse). The crater depth is measured with a confocal laser-scanning microscope (VK-X250/260, Keyence) with a depth resolution of $0.5 \mathrm{~nm}$.

Figures 1(a), 1(b), and 1(c) show examples of doublepulse irradiated surfaces imaged with an SEM at a magnification of $3000 \times$. The fluences are $(140,140)$ and the pulse intervals are $1 \mathrm{ps}, 10 \mathrm{ps}$, and $1280 \mathrm{ps}$. The morphologies of the nanostructures clearly change as the time interval is increased. The structure in Fig. 1(b) shows the clearest periodicity. To evaluate the clarity of periodicity quantitatively, we apply a Fourier transform to each image (Fig. 1(d)). The sharpness of the spectrum corresponds to the clarity of periodicity. Here, we define the peak to be the grating "interspace." In Fig. 1(d), only the solid line shows a clear peak, indicating the existence of a periodic structure with an interspace of around $600 \mathrm{~nm}$.

Figures 2(a) and 2(b) show how the Fourier spectra change when the interval is varied $(0.16-1280 \mathrm{ps})$, for the fluences $(140,140)$ and $(70,140)$, respectively. In Fig. 2(a), for intervals of less than $10 \mathrm{ps}$, the peak intensities of the spectrum are relatively low. At around 10 ps the peak intensity is a maximum, and above 10 ps the spectrum becomes flat. This means that clear periodic structures are formed only when the interval is about 10 ps. In contrast, the spectrum in Fig. 2(b) shows a clear peak for all intervals with a maximum for intervals of several hundreds of picoseconds. The interspaces are constant around $600 \mathrm{~nm}$ regardless of the interval. The difference between Figs. 2(a) and 2(b) is due to the choice of the first pulse's fluence $F_{1}$. For Fig. 2(a), $F_{1}$ is higher than the threshold fluence $F_{\text {th }}$, so the first pulse would create surface plasma. For Fig. 2(b), in contrast, $F_{1}$ is lower than $F_{\text {th }}$, so the first pulse would not create surface plasma. Therefore, the formation of clear periodic structures would be disturbed by the pre-formed surface plasma for Fig. 2(a) but would not be disturbed for Fig. 2(b). This suggests that the first pulse irradiation certainly affects the structures induced by the second pulse.

Figures 3(a) and 3(b) show the dependence of ablation rate on interval $\Delta t$ for the fluences $(140,140)$ and $(70,140)$, respectively. The shape of the dependence is roughly independent of fluence: the ablation rate decreases as the interval
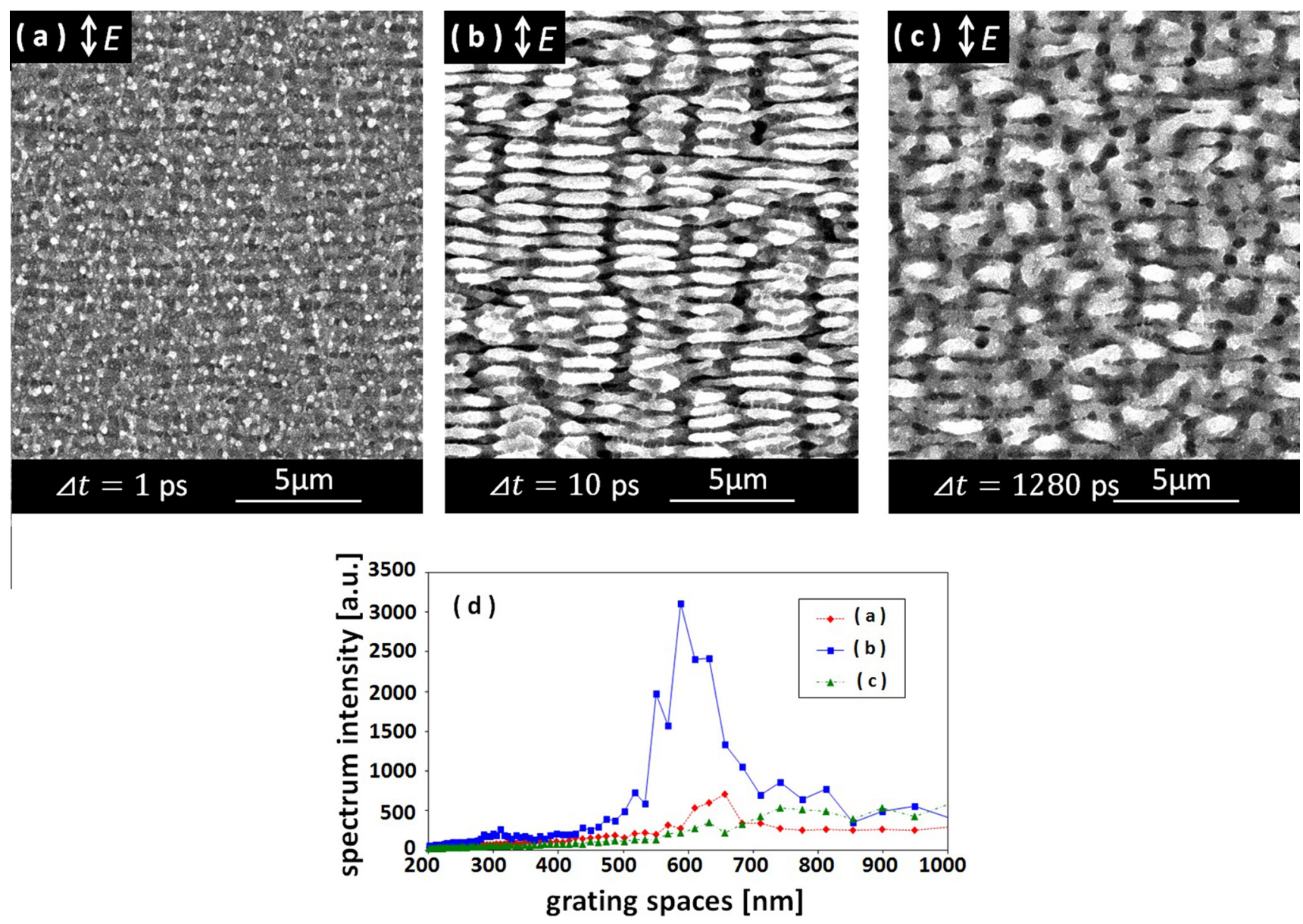

FIG. 1. Examples of SEM images with intervals $\Delta t$ of (a) $1 \mathrm{ps}$, (b) $10 \mathrm{ps}$, and (c) $1280 \mathrm{ps}$ (fluences $(140,140), 50$ pairs). (d) 1D Fourier spectra obtained from $(a),(b)$, and (c). Each graph shows spectrum intensity versus grating spaces. The direction of LIPSS growth is perpendicular to the laser polarization $(E)$. 

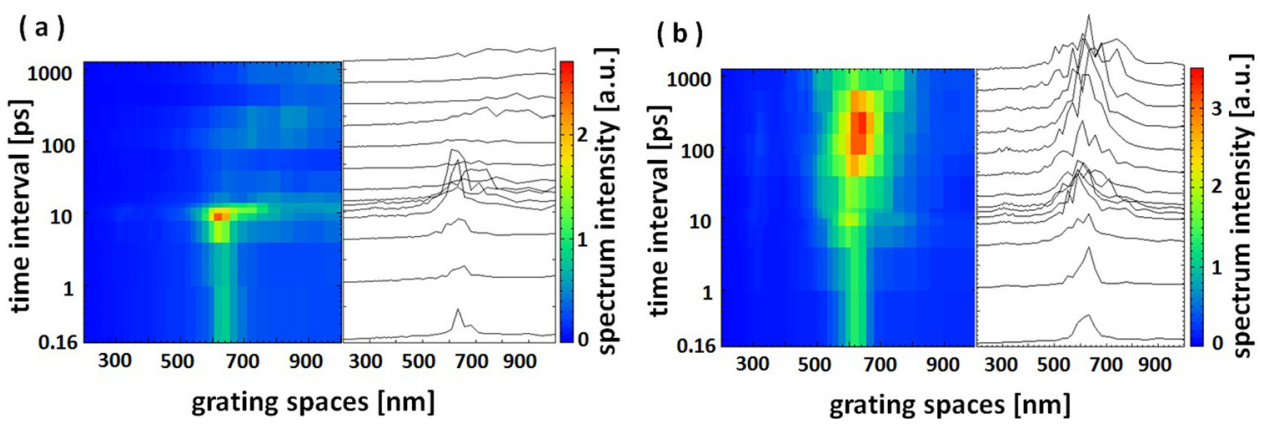

FIG. 2. Time evolution of spectrum intensity for fluences (a) $(140,140)$ and (b) $(70,140)$. Intervals are shown on a logarithmic scale. Spectrum intensities in the color maps are indicated by the color bars on the right-hand sides of the figures.

increases for $\Delta t<20 \mathrm{ps}$, does not change much for $20 \mathrm{ps}<\Delta t<300 \mathrm{ps}$, and increases for $\Delta t>300 \mathrm{ps}$.

For the fluences $(140,140)$, as shown in Fig. 3(a), the maximum ablation rate is $19.5 \mathrm{~nm}$ for $\Delta t=0.16 \mathrm{ps}$, which is almost as much as that $(20.1 \mathrm{~nm})$ for single-pulse irradiation of $280 \mathrm{~mJ} / \mathrm{cm}^{2}$. The minimum is $2.0 \mathrm{~nm}$ for $\Delta t=320 \mathrm{ps}$, which is about a quarter of that $(8.3 \mathrm{~nm})$ for $140 \mathrm{~mJ} / \mathrm{cm}^{2}$ single-pulse irradiation. This indicates that the first pulse modifies the surface to some extent, reducing ablation by the second pulse. A similar phenomenon has previously been reported, ${ }^{13}$ but with only a $60 \%$ reduction in ablation rate by double-pulse irradiation.

For the fluences $(70,140)$ (Fig. 3(b)), the maximum and minimum ablation rates are $15.5 \mathrm{~nm}$ for $\Delta t=0.16 \mathrm{ps}$ and $4.4 \mathrm{~nm}$ for $\Delta t=80 \mathrm{ps}$, respectively. The maximum is as much as that $(17.1 \mathrm{~nm})$ for a single pulse of $210 \mathrm{~mJ} / \mathrm{cm}^{2}$; the minimum $(4.4 \mathrm{~nm})$ is half of that for a $140 \mathrm{~mJ} / \mathrm{cm}^{2}$ single pulse. Thus, the first pulse modifies the surface to suppress ablation even if its fluence is below the ablation threshold. By reducing the fluence of the first pulse from 140 to $70 \mathrm{~mJ} /$ $\mathrm{cm}^{2}$, the ablation could be increased (from a quarter to a half of that for single pulse), giving an ablation rate comparable with that at the threshold fluence for LIPSS formation with a single pulse. ${ }^{4}$ This demonstrates that double-pulse irradiation can control both the ablation rate and LIPSS formation by adjusting the fluence of the first pulse and the interval between pulses.

Figure 3 shows that double-pulse irradiation holds promise as an advanced fabrication technique beyond single-pulse irradiation. For example, it may be possible to ablate an area much smaller than the diffraction limit by reducing the ablation rate with local double-pulse irradiation. In addition, controlling the periodicity and depth of
LIPSS would be significant for improving biocompatibility with various kinds of living cells.

The mechanism of the phenomena mentioned above is under debate, and a plasma shielding model has been proposed by Semerok and Dutouquet. ${ }^{17}$ In this model, the plasma produced by the first pulse shields the second pulse and prevents the energy of the second pulse from being transmitted to the target after a delay of 1 ps. Our experimental results show a reduction after several picoseconds, but two results cannot be explained by this model. First, the ablation rate for the fluences $(70,140)$ decreases even though the first pulse would not produce a plasma sufficient to shield the second pulse because the first pulse's fluence is smaller than the threshold fluence. This suggests that there is another mechanism that decreases the ablation rate even in the absence of an expanding laser-produced plasma. Second, the ablation rate for a double pulse should be greater than or equal to that of a single pulse. However, we obtain ablation rates lower than those for a single pulse. These two points cannot be understood with the plasma shielding model, and it is necessary to understand these phenomena for double-pulse irradiation to be applied in future laser nano-processing.

We have demonstrated that double-pulse irradiation is useful for producing femtosecond LIPSS with a lower ablation rate than that produced by single-pulse irradiation. The experimental results suggest that the first pulse influences the surface, suppressing ablation by the following pulse even though no laser plasma is produced on the surface, and that this influence continues for rather a long time (several hundred picoseconds). More study is necessary to understand the function of the first pulse, but double-pulse irradiation certainly has significant potential for future applications of laser nano-processing.
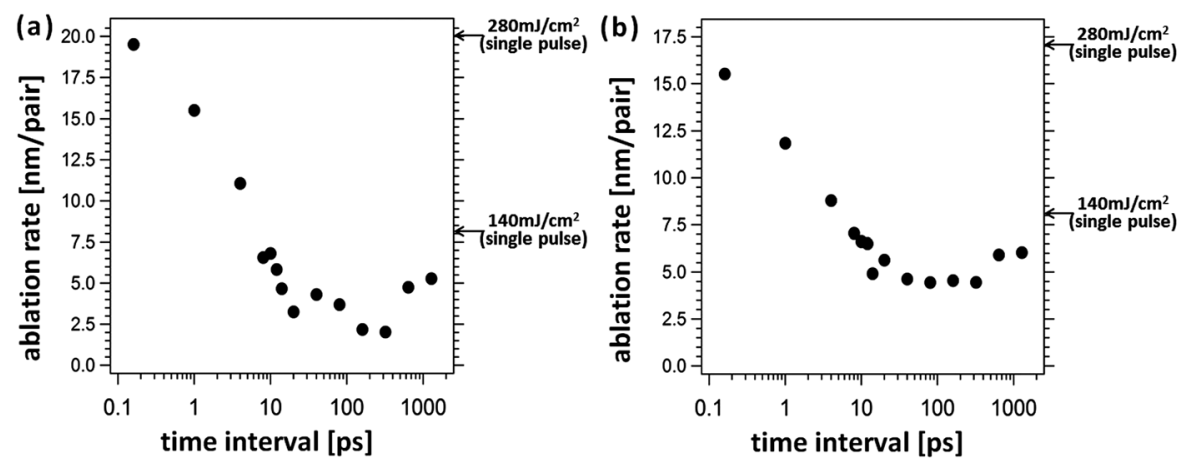

FIG. 3. Dependence of ablation rate on time interval for fluences (a) (140, $140)$ and (b) $(70,140)$. 
We thank K. Nishida and Y. Tsujii for their assistance with scanning electron microscopy and confocal laser scanning microscopy. This research was financially supported by JSPS KAKENHI (Grant No. 16K06745) and by the Amada Foundation (AF-2011215).

${ }^{1}$ J. Bonse, J. Krüger, S. Höhm, and A. Rosenfeld, J. Laser Appl. 24, 042006 (2012).

${ }^{2}$ R. Buividas, M. Mikutis, and S. Juodkazis, Prog. Quantum Electron. 38, 119 (2014).

${ }^{3}$ R. Buividas, T. Kudrius, R. Šliupas, L. Rosa, G. Šlekys, S. Bagdonas, R. Rotomoskis, and S. Juodkazis, Proc. SPIE 7376, 737602 (2010).

${ }^{4}$ M. Hashida, Y. Ikuta, Y. Miyasaka, S. Tokita, and S. Sakabe, Appl. Phys. Lett. 102, 174106 (2013).

${ }^{5}$ T. Kato and N. Abe, Rev. Laser Eng. 37, 510 (2009).

${ }^{6}$ N. Yasumaru, K. Miyazaki, and J. Kiuchi, Appl. Surf. Sci. 254, 2364 (2008).

${ }^{7}$ B. Wo, M. Zhou, J. Li, X. Ye, G. Li, and L. Cai, Appl. Surf. Sci. 256, 61 (2009).
${ }^{8}$ T. Shinonaga, M. Tsukamoto, T. Kawa, P. Chen, A. Nagai, and T. Hanawa, Appl. Phys. B 119, 493 (2015).

${ }^{9}$ R.-A. Barb, C. Hrelescu, L. Dong, J. Heitz, J. Siegel, P. Slepicka, V. Vosmanska, V. Svorcik, B. Magnus, R. Marksteiner, M. Schernthaner, and K. Groschner, Appl. Phys. A 117, 295 (2014).

${ }^{10}$ A. Y. Vorobyev and C. Guo, Appl. Phys. Lett. 92, 041914 (2008).

${ }^{11}$ S. Sakabe, M. Hashida, S. Tokita, S. Namba, and K. Okamuro, Phys. Rev. B 79, 033409 (2009).

${ }^{12}$ K. Okamuro, M. Hashida, Y. Miyasaka, Y. Ikuta, S. Tokita, and S. Sakabe, Phys. Rev. B 82, 165417 (2010).

${ }^{13}$ D. E. Roberts, A. du Plessis, and L. R. Botha, Appl. Surf. Sci. 256, 1784 (2010).

${ }^{14}$ S. Höhm, A. Rosenfeld, J. Krüger, and J. Bonse, J. Appl. Phys. 112, 014901 (2012).

${ }^{15}$ S. Höhm, M. Herzlieb, A. Rosenfeld, J. Krüger, and J. Bonse, Opt. Express 23, 61 (2015).

${ }^{16}$ S. Höhm, A. Rosenfeld, J. Krüger, and J. Bonse, Opt. Express 23, 25959 (2015).

${ }^{17}$ A. Semerok and C. Dutouquet, Thin Solid Films 453-454, 501 (2004). 